This item was submitted to Loughborough's Research Repository by the author.

Items in Figshare are protected by copyright, with all rights reserved, unless otherwise indicated.

\title{
Influence of strain gradients on lattice rotation in nano-indentation experiments: a numerical study
}

PLEASE CITE THE PUBLISHED VERSION

http://dx.doi.org/10.1016/j.msea.2014.04.033

\section{PUBLISHER}

(C) Elsevier B.V.

\section{VERSION}

AM (Accepted Manuscript)

\section{PUBLISHER STATEMENT}

This work is made available according to the conditions of the Creative Commons Attribution-NonCommercialNoDerivatives 4.0 International (CC BY-NC-ND 4.0) licence. Full details of this licence are available at: https://creativecommons.org/licenses/by-nc-nd/4.0/

\section{LICENCE}

CC BY-NC-ND 4.0

\section{REPOSITORY RECORD}

Demiral, Murat, Anish Roy, Tamer El Sayed, and Vadim V. Silberschmidt. 2019. "Influence of Strain Gradients on Lattice Rotation in Nano-indentation Experiments: A Numerical Study”. figshare. https://hdl.handle.net/2134/16621. 


\title{
Influence of strain gradients on lattice rotation in nano-indentation experiments: A numerical study
}

\author{
Murat Demiral $^{1}$, Anish Roy* ${ }^{2}$, Tamer El Sayed ${ }^{1}$, Vadim V. Silberschmidt ${ }^{2}$ \\ ${ }^{1}$ Computational Solid Mechanics Laboratory, Division of Physical Sciences and \\ Engineering, King Abdullah University of Science and Technology (KAUST), \\ Saudi Arabia \\ ${ }^{2}$ Wolfson School of Mechanical and Manufacturing Engineering, Loughborough \\ University, LE11 3TU UK \\ *Corresponding author. Tel.: +44 1509227 566; E-mail: A.Roy3@lboro.ac.uk
}

\begin{abstract}
In this paper the texture evolution in nano-indentation experiments was investigated numerically. To achieve this, a three-dimensional implicit finiteelement model incorporating a strain-gradient crystal-plasticity theory was developed to represent accurately the deformation of a body-centred cubic metallic material. A hardening model was implemented to account for strain hardening of the involved slip systems. The surface topography around indents in different crystallographic orientations was compared to corresponding lattice rotations. The influence of strain gradients on the prediction of lattice rotations in nanoindentation is critically assessed.
\end{abstract}

Keywords: Lattice rotation; Pile-up; Indentation; Strain-gradient crystal plasticity; Ti alloy 


\section{Introduction and motivation}

Indentation is an experimental method widely used to characterize the mechanical response of small volumes of materials [1-3]. Although indentation experiments are easy to perform, interpreting the obtained data is a challenge due to the inherent heterogeneity of the deformation fields, complex stress/strain distributions in the tested material and the non-trivial contact conditions during the process. Consequently, numerical simulation techniques are frequently used to study the underlying mechanics in indentation experiments. For instance, deformationinduced lattice rotations below an indent have attracted attention as there exists a close connection between crystallographic shear, the main mechanism governing the deformation, and the resulting lattice spin [4].

Some studies have attempted to characterise the observed phenomena, with the use of different techniques such as the non-destructive 3D synchrotron diffraction method [5], 3D electron backscattered diffraction (EBSD) [4] and transmission electron microscopy (TEM) [6-7].

In this regard, a limited number of numerical studies attempted to analyse physical deformation mechanisms leading to lattice rotations [8-9]. Wang et al. [10] demonstrated lattice rotations for a single crystal of $\mathrm{Cu}$ with different orientations using a 3D elastic-viscoplastic crystal-plasticity (CP) finite-element (FE) method. Zaafarani and co-workers proposed a physically based crystal-plasticity model based on dislocation-rate formulations to explain the potential reasons for deformation-induced patterns consisting of multiple narrow zones with alternating crystalline rotations [11]. However, the model consistently overestimated the extent of lattice rotations in the experiment.

Strain-gradient plasticity theories [12-16] that account for the effects of geometrically necessary dislocations (GNDs) [17] on plastic flow had some success in explaining size effects observed in torsion [18], bending [19] and 
indentation [20-22]. In this paper, a 3D non-local elastic-viscoplastic crystalplasticity finite-element model for the nano-indentation of Ti-15V-3Al-3Sn-3Cr (Ti-15-3-3-3) is developed to demonstrate the influence of strain gradients on the reorientation of the crystalline lattice and resulting deformation patterns. The relationship between the anisotropy of surface profiles around nanoindents and local texture changes is studied.

This paper is organized as follows: a brief self-contained description of constitutive equations of the strain-gradient crystal-plasticity theory is presented in Section 2. Details of the developed finite-element model of nano-indentation are presented in Section 3. Section 4 demonstrates the predictive capabilities of the model for lattice rotations and surface profiles for different crystallographic orientations. We finally offer concluding remarks in Section 5.

\section{Theory}

An enhanced modelling scheme for a strain-gradient crystal-plasticity (EMSGCP) theory proposed by Demiral [23] was used in the simulations. Below, the constitutive relations of the theory are summarized.

The deformation gradient $\boldsymbol{F}$ is decomposed multiplicatively into elastic $\left(\boldsymbol{F}^{\mathrm{e}}\right)$ and plastic $\left(\boldsymbol{F}^{\mathrm{p}}\right)$ parts:

$$
\boldsymbol{F}=\boldsymbol{F}^{\mathrm{e}} \boldsymbol{F}^{\mathrm{p}} .
$$

$\boldsymbol{F}^{\mathrm{p}}$ evolves according to the flow rule

$$
\dot{\boldsymbol{F}}^{\mathrm{p}}=\boldsymbol{L}^{\mathrm{p}} \boldsymbol{F}^{\mathrm{p}}
$$

where the plastic velocity gradient $\boldsymbol{L}^{\mathrm{p}}$ corresponds to

$$
\boldsymbol{L}^{\mathrm{p}}=\sum_{\alpha=1}^{N} \dot{\gamma}^{\alpha} \boldsymbol{s}^{\alpha} \otimes \boldsymbol{m}^{\alpha} .
$$

In Equation (3), $\dot{\gamma}^{\alpha}$ is the shearing rate on the slip system $\alpha$, which is represented by the slip direction $\boldsymbol{s}^{\alpha}$ and the slip plane normal $\boldsymbol{m}^{\alpha}$. We choose a power-law representation for $\dot{\gamma}^{\alpha}$, as 


$$
\dot{\gamma}^{\alpha}=\dot{\gamma}_{0}^{\alpha} \operatorname{sgn}\left(\tau^{\alpha}\right)\left|\frac{\tau^{\alpha}}{g_{T}^{\alpha}}\right|^{n},
$$

where $\dot{\gamma}_{0}^{\alpha}$ is the reference strain rate, $n$ is the macroscopic rate-sensitivity parameter, $\tau^{\alpha}$ is the resolved shear stress, $g_{T}^{\alpha}$ is the strength of the slip system $\alpha$ at the current time, and $\operatorname{sgn}(*)$ is the signum function of *.

In the EMSGCP theory, the initial strength of slip systems, i.e. the critical resolved shear stress (CRSS), is governed by pre-existing GNDs in the workpiece together with statistically stored dislocations (SSDs) (Eq. (5)), i.e. $g_{T}^{\alpha} t_{k=0}=g_{S}^{\alpha} t_{t=0}+g_{G}^{\alpha} t_{t=0}$. Here, a subscript $G$ indicates GNDs and $S$ implies SSDs. In this theory, $g_{S}^{\alpha} t_{t=0}$ and $\left.g_{G}^{\alpha}\right|_{t=0}$ were linked with initial SSD $\left(\rho_{S}^{\alpha} t_{t=0}\right)$ and GND $\left(\rho_{G}^{\alpha} t_{t=0}\right)$ densities as $\left.g_{S}^{\alpha}\right|_{t=0}=K \sqrt{\left.\rho_{S}^{\alpha}\right|_{t=0}},\left.g_{G}^{\alpha}\right|_{t=0}=K \sqrt{\left.\rho\right|_{t=0}(\bar{S} / \bar{V})^{2}}$ via the constant, $K$, similar to the Taylor relation. The GND density term was expressed as a function of the normalized surface-to-volume $(\bar{S} / \bar{V}$ ) ratio (hence, dimensionless) for the component under study [24].

The evolution of slip resistance during loading is the result of hardening due to the SSDs $\left(\Delta g_{s}^{\alpha}\right)$ and GNDs $\left(\Delta g_{G}^{\alpha}\right)$ on the slip system:

$$
\begin{gathered}
g_{T}^{\alpha}=\left.g_{S}^{\alpha}\right|_{t=0}+\left.g_{G}^{\alpha}\right|_{t=0}+\sqrt{\left(\Delta g_{S}^{\alpha}\right)^{2}+\left(\Delta g_{G}^{\alpha}\right)^{2}} \\
\Delta g_{S}^{\alpha}=\sum_{\beta=1}^{N} h_{\alpha \beta} \Delta \gamma^{\beta}, \Delta g_{G}^{\alpha}=\alpha_{\mathrm{T}} \mu_{\mathrm{s}} \sqrt{b n_{G}^{\alpha}} .
\end{gathered}
$$

Here, $h_{\alpha \beta}, \alpha_{\mathrm{T}}, \mu_{\mathrm{s}}, b$ and $n_{G}^{\alpha}$ corresponds to the slip-hardening modulus, the Taylor coefficient, the shear modulus, the Burgers vector and the effective density of geometrically necessary dislocations, respectively. The hardening model proposed by Peirce et al. [25] is used to represent $h_{\alpha \beta}$, as follows: 


$$
h_{\alpha \alpha}=h_{0} \operatorname{sech}^{2}\left|\frac{h_{0} \tilde{\gamma}}{\left.g_{T}^{\alpha}\right|_{\text {sat }}-\left.g_{T}^{\alpha}\right|_{t=0}}\right|, h_{\alpha \beta}=q h_{\alpha \alpha}(\alpha \neq \beta), \tilde{\gamma}=\sum_{\alpha} \int_{0}^{t}\left|\dot{\gamma}^{\alpha}\right| d t \text {, }
$$

where $h_{0}$ is the initial hardening parameter, $\left.g_{T}^{\alpha}\right|_{\text {sat }}$ is the saturation stress of the slip system $\alpha, q$ is the latent hardening ratio, which is assumed to be 1 , and $\tilde{\gamma}$ is the Taylor cumulative shear strain on all slip systems. The effective GND density $\left(n_{G}^{\alpha}\right)$ is given by

$$
n_{G}^{\alpha}=\left|\boldsymbol{m}^{\alpha} \times \sum_{\beta} s^{\alpha \beta} \nabla \gamma^{\beta} \times \boldsymbol{m}^{\beta}\right|,
$$

where $s^{\alpha \beta}=\boldsymbol{s}^{\alpha} . \boldsymbol{s}^{\beta}$ and $\nabla \gamma^{\beta}$ is the gradient of shear strain in each slip system. To calculate $\nabla \gamma^{\beta}$ the scheme proposed in Demiral et al. [24] is followed. The model was implemented in the implicit finite-element code ABAQUS/Standard using the user-defined material subroutine (UMAT). Relevant details can be found in [21,24,26-27].

It should be noted that as the $\bar{S} / \bar{V}$ ratio is negligibly small in nano-indentation samples, the CRSS value of slip systems depends only on SSDs. Therefore, for the nano-indentation test, the EMSGCP theory naturally reduces to the mechanismbased strain-gradient crystal-plasticity theory proposed by Han et al. [28]. Note that in the classical CP theory the contribution from both incipient and evolving GNDs is not accounted for, i.e. instantaneous strength of the slip system is given by $g_{T}^{\alpha}=\left.g_{S}^{\alpha}\right|_{t=0}+\Delta g_{S}^{\alpha}$.

Here, a $\beta$-Ti alloy with a b.c.c. crystalline structure is studied. In the following simulations only the $\{112\}<111>$ slip system (Table 1 ) was considered [24].

Table 1

\section{Finite-element modelling of nano-indentation}


A FE model of the indentation experiment was developed [24]. Dimensions of the workpiece sample used in the FE model were $10 \mu \mathrm{m} \times 10 \mu \mathrm{m} \times 6 \mu \mathrm{m}$. Eight-node linear brick elements (C3D8) were used to discretise the sample. A finer mesh with a minimum element size of $100 \mathrm{~nm}$ was used near the indenter tip as the strain gradients are typically the highest in the vicinity of the indenter. A conical indenter with $\theta=90^{\circ}$ and a tip radius of $1.0 \mu \mathrm{m}$ was modelled as a rigid body. The indenter was displaced in the negative y-direction with a maximum indentation depth of 375 $\mathrm{nm}$ followed by complete unloading. The bottom face of the workpiece was constrained in all directions, its faces with normals in the $\mathrm{x}$ - and $\mathrm{z}$ - directions were constrained, respectively. Contact between the indenter and workpiece was assumed to follow Coulomb’s friction law with $\mu=0.05$ [23].

The material parameters used in the simulations are listed in Table 2. These are based on their exhaustive calibration by matching the surface profile of the indented surface along a path with the experimental data (Fig. 1). It must be noted that as the experimentally obtained surface profiles were not scanned with an ideally sharp AFM indenter tip, the holes were imaged sharper. This effect explains the differences in the experimentally and numerically obtained surface profiles in (Fig. 1) [30]. The pertinent details are presented elsewhere [24].

Table 2

\section{Fig. 1}

\section{Results and discussion}

In this section, the results of FE simulations of the nano-indentation for three different crystallographic orientations, viz. (100), (101) and (111), are presented with the corresponding lattice rotations and profiles of surface pile-ups. Crystalline reorientation around indents for the crystallographic orientations studied is shown in Fig. 2. Lattice rotations about the $\mathrm{x}$ - and $\mathrm{y}$ - axes (defined for each orientation studied) are compared. It was observed that there exists a symmetry in the texture evolution of the indented (010) surface, where the $\mathrm{x}$ - and $\mathrm{y}$ - axes correspond to [1 0 
1] and [1 0 -1] directions, respectively. The magnitudes of maximum rotation were almost identical in both cases, with an angle of $\sim \pm 37^{\circ}$. Symmetry was also observed for the indented (101) surface, where lattice rotations about the x-axis were more pronounced than that about the y-axis. For the (111) surface, the symmetry about the $y$-axis was observed with a lattice rotation of $\pm 27^{\circ}$. However, this symmetry was broken, about the x-axis.

Fig. 2

To understand the reason for the observed symmetries in induced lattice rotations, the corresponding crystallographic shear strains for different crystal orientations were investigated. In indentation, the material's translation occurs along the intersection vectors of the primary slip systems with the indented surface [31], leading, in turn, to different types of symmetries. For instance, indentation of the (001) oriented single crystal demonstrate a 4-fold symmetry, while crystals with (011) and (111) orientations show a 2-fold and 3-fold symmetries, respectively (Fig. 3) $[10,31]$. The differences in pile-up patterns demonstrate strong crystallographic anisotropy of the out-of plane displacements in the vicinity of the indents.

For instance, Fig. 3(a) suggests that the surface profile for the indented (010) surface is symmetric with respect to both [ $\left.\begin{array}{lll}1 & 0 & 1\end{array}\right]$ and $\left[\begin{array}{lll}1 & 0 & -1\end{array}\right]$ axes and the maximum pile-up height is almost identical at four peak points. This, in fact, explains the symmetries of lattice spins with respect to the respective axes in Fig. 2(a). The maximum pile-up regions in the indented (101) surface occurred about the $\left[\begin{array}{lll}-1 & 0 & 1\end{array}\right]$ axis rather than [0 -10$]$ axis (Fig. 3(b)) which ultimately resulted in higher lattice rotations of $\sim 46^{\circ}$ to $48^{\circ}$ about the [0 -10$]$ axis (Fig. 2(b)). On the other hand, the pile-ups observed on the indented (111) surface are symmetric about the [1 -2 1] axis (Fig. 3(c)); thus, corresponding lattice rotations were symmetric (Fig. 2(c)) when compared to [-1 0 ll] axis, where both the pile-ups and lattice rotations are not symmetric. It was observed that higher lattice rotations were observed on the lower side of [ $\left[\begin{array}{lll}-1 & 0 & 1\end{array}\right]$ axis, where the volume of the material experienced piling-up was higher compared to its upper side. These observations 
suggest that there exists a strong relationship between the patterns of induced lattice rotations and the indents' pile-up profiles.

It should be noted that the maximum pile-up height is the largest for the indented ( 0 10 ) surface with a value of $80 \mathrm{~nm}$, and smallest for the indented (llll) surface (Fig. 3). The reason for this difference can be explained by the total number of active slip systems during deformation, which, in turn, leads to the occurrence of cross slips and, consequently, an increase in the pile-up height [32]. Our FE simulations demonstrated that all the slip systems were active for the indented ( $\begin{array}{ll}0 & 1\end{array}$ 0 ) surface, whereas the total number of 8 and 10 slip systems were active for the indented (111) and (101) surfaces, respectively.

Fig. 3

Next, the lattice rotations underneath the indent were investigated. Fig. 4(a) shows the net orientation changes with reference to the starting orientation of the crystal at the cross-section of the $\left(\begin{array}{lll}-1 & 0 & 1\end{array}\right)$ plane for the indented $(010)$ surface at complete unloading. The distribution of deformation-induced lattice rotations shows a double-well pattern with a change in local rotational fields varying between $\pm 20^{\circ}$ except for the regions, where high lattice reorientation occurred. The (-1 01 1) plane for the indented (010) surface can be divided into three sections. Region $A$ is bounded by the points A1-A2-A4-A3, region B is bounded by A1-A2-C2-C1 and A3-A4-C4-C3, and the remainder of the domain shown in Fig. 4(a) is region C. Lattice rotations in regions $\mathrm{A}$ and $\mathrm{C}$ are observed to be comparatively lower than those observed in region $\mathrm{B}$.

\section{Fig. 4}

The distribution of texture evolution at different loading steps along the paths C-D and E-F in Fig. 4 is shown in Fig. 5. It should be noted that the chosen paths coincide with the edges of deformed finite elements in the mesh. The plot indicates that at full loading, the orientation change in regions $A$ and $C$ is less than $\pm 6^{\circ}$, whereas in region $B$, the lattice orientation changes by greater magnitudes. Note that the current model has no back-stress term and this may influence the unloading 
results but the deviation from an orientation change of $\pm 6^{\circ}$ is not expected to be significant.



(a)

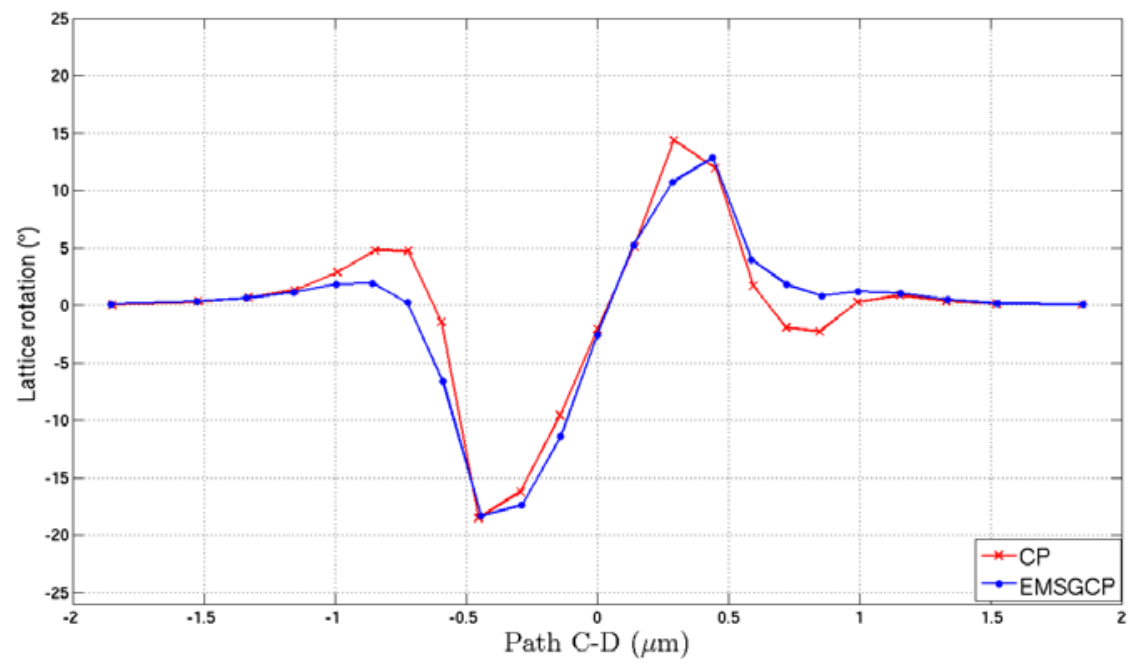

(b) 




(c)

Previous Fig. 6a will be here

(d)

Previous Fig. 6b will be here

(e)

Fig. 5: Lattice rotation along path C-D at half loading $(t=0.5 \mathrm{~s})$ (a), three-fourths loading ( $t=0.75 \mathrm{~s}$ ) (b), full loading ( $t=1.0 \mathrm{~s})$ (c), complete unloading ( $t=1.0 \mathrm{~s})$ (d) and along path E-F at complete unloading (e) (Fig. 4) in (-1 0 1) plane of (010) orientation obtained with FE simulations using CP and EMSGCP theories

FE simulations were also performed with the CP constitutive laws to compare and contrast the influence of strain-gradient effects on crystalline reorientation. Fig. 4(b) shows the respective lattice rotations of the crystal at the cross-section of $\left(\begin{array}{lll}-1 & 0 & 1\end{array}\right)$ plane for the indented (010) surface, with the values along the paths C-D and E-F at different loading steps plotted in Fig. 5. It was found that at complete unloading the local rotational fields were closer to those obtained with the EMSGCP constitutive laws at some regions such as at positions 2 and 3 on path C-D and at position 6 on path E-F. However, these values were overestimated significantly at 
positions 1 and 4 on path C-D and at position 5 on path E-F by a factor of 3.3, 5.1 and 2.7, respectively (Table 3). The reason for the discrepancy arises from the fact that GNDs are not accounted for in the CP constitutive equations. In an indentation experiment, rapid spatial changes can occur in the local texture due to imposed boundary conditions. The resulting mismatch in the lattice spin for a material point can be accommodated by GNDs. As this is accounted for in the EMSGCP theory, the predicted orientation change is less pronounced in comparison to that for the CP theory (Fig 5(a)-(c)). With an increase in indenter penetration, strain gradients became more significant and, as a result, the texture evolution predicted with the EMSGCP theory at some spatial positions (i.e. positions 1 and 4) start to diverge from that for the CP theory. These observations suggest a possible reason for the overestimation of experimentally obtained texture evolutions using the CP theory in Zaafarani et al. $[4,11]$.

\section{Table 3}

In Fig. 1 the surface profiles obtained by the CP and the EMSGCP theories were compared. It was observed that the maximum pile-up height after unloading was overestimated by $32 \%$ using the CP theory whereas an accurate match with the experiment was obtained using the EMSGCP theory. In the absence of strain gradients in the CP theory, the material's cumulative strength is predicted to be lower, leading to a conventional plastic response of the material beyond the yield point. As a result, the strain due to elastic spring back decreased and, consequently, the pile-up height increased due to higher plastic deformation [24].

One of the advantages of the developed FE model is that it provides information about the spatial 3D distribution of the individual shear strains on all the slip systems, which cannot be obtained by any experimental or analytical techniques. The accumulated shear strain values, at complete unloading, from the CP and EMSGCP theories for each of the 12 slip systems at positions 1 and 2 on path C-D are demonstrated in Fig. 6. 
First, it was observed that the total residual shear-strain values on all the slip systems were larger in the prediction based on the CP theory than those based on the EMSGCP theory. This confirmed that the elastic spring back as predicted from CP was lower in magnitude.

Secondly, the number of active slip systems was found to be sensitive to both the spatial position and the constitutive law used. A strain of 0.01 was assumed as the threshold value for a slip system to be active. For instance, at position 1, while the number of active slip systems were found to be only 6 using the CP theory, it was 8 when the EMSGCP theory was used, whereas, both theories predicted 10 active slip systems at position 2.

Fig. 6

Thirdly, it was observed that the relative contributions of different slip systems to the overall deformation were different for different theories. For instance, at Point 1 at the end of loading (Table 4), the CP theory predicts that the first and second slip systems accommodates $80 \%$ of the overall shear in the component. In contrast, in the EMSGCP model the contribution of the most active slip systems, i.e. slip systems 1 and 2, accounts for $\sim 53 \%$ of the overall shear in the system. It was noticed that more slip systems contribute to the overall deformation in EMSGCP when compared to CP-based predictions. This demonstrates that strain-gradients heavily influence individual slip system activity, which sometimes manifest in noticeable difference in spatial deformations. Consequently, there are locations where the overall slip activity seems to normalise, demonstrating identical response (e.g. Point 2).

Table 4: Shear-strain magnitudes and their relative values (bold; in \%) for different slip systems at various positions along path C-D (see Fig. 4) at different loading stages in (-1 0 1) plane of (010) orientation

\begin{tabular}{cccccccc}
\hline & \multicolumn{2}{c}{$\begin{array}{c}\text { Point } 1 \text { at half } \\
\text { loading }\end{array}$} & \multicolumn{2}{c}{$\begin{array}{c}\text { Point } 1 \text { at full } \\
\text { loading }\end{array}$} & \multicolumn{2}{c}{$\begin{array}{c}\text { Point } 2 \text { at full } \\
\text { loading }\end{array}$} \\
\hline $\begin{array}{c}\text { Slip } \\
\text { system }\end{array}$ & CP & EMSGCP & CP & EMSGCP & CP & EMSGCP \\
\hline 1 & 0.0404 & 0.0333 & 0.2670 & 0.1474 & 0.0002 & 0.0005 \\
\hline
\end{tabular}




\begin{tabular}{ccccccc}
\hline & $\mathbf{3 6 . 3}$ & $\mathbf{3 6 . 5}$ & $\mathbf{4 0 . 3}$ & $\mathbf{2 6 . 5}$ & $\mathbf{0 . 0}$ & $\mathbf{0 . 0}$ \\
\hline \multirow{2}{*}{2} & 0.0387 & 0.0332 & 0.2630 & 0.1480 & 0.0003 & 0.0005 \\
& $\mathbf{3 4 . 8}$ & $\mathbf{3 5 . 4}$ & $\mathbf{3 9 . 7}$ & $\mathbf{2 6 . 6}$ & $\mathbf{0 . 0}$ & $\mathbf{0 . 0}$ \\
\hline \multirow{2}{*}{3} & 0.0 & 0.0 & 0.0020 & 0.0058 & 0.0910 & 0.0566 \\
& $\mathbf{0 . 0}$ & $\mathbf{0 . 0}$ & $\mathbf{0 . 3}$ & $\mathbf{1 . 0}$ & $\mathbf{5 . 7}$ & $\mathbf{4 . 5}$ \\
\hline \multirow{2}{*}{4} & 0.0 & 0.0 & 0.0015 & 0.0075 & 0.0939 & 0.0654 \\
& $\mathbf{0 . 0}$ & $\mathbf{0 . 0}$ & $\mathbf{0 . 2}$ & $\mathbf{1 . 3}$ & $\mathbf{5 . 9}$ & $\mathbf{5 . 2}$ \\
\hline \multirow{2}{*}{5} & 0.0 & 0.0 & 0.0 & 0.0001 & 0.3485 & 0.2690 \\
& $\mathbf{0 . 0}$ & $\mathbf{0 . 0}$ & $\mathbf{0 . 0}$ & $\mathbf{0 . 0}$ & $\mathbf{2 1 . 8}$ & $\mathbf{2 1 . 4}$ \\
\hline \multirow{2}{*}{6} & 0.0 & 0.0 & 0.0 & 0.0001 & 0.3310 & 0.2641 \\
& $\mathbf{0 . 0}$ & $\mathbf{0 . 0}$ & $\mathbf{0 . 0}$ & $\mathbf{0 . 0}$ & $\mathbf{2 0 . 7}$ & $\mathbf{2 1 . 0}$ \\
\hline \multirow{2}{*}{7} & 0.0020 & 0.0004 & 0.0186 & 0.0179 & 0.0169 & 0.0395 \\
& $\mathbf{1 . 8}$ & $\mathbf{0 . 5}$ & $\mathbf{2 . 8}$ & $\mathbf{3 . 2}$ & $\mathbf{1 . 1}$ & $\mathbf{3 . 1}$ \\
\hline \multirow{2}{*}{8} & 0.0010 & 0.0006 & 0.0098 & 0.0150 & 0.0183 & 0.0370 \\
& $\mathbf{0 . 9}$ & $\mathbf{0 . 6}$ & $\mathbf{1 . 5}$ & $\mathbf{2 . 7}$ & $\mathbf{1 . 1}$ & $\mathbf{2 . 9}$ \\
\hline \multirow{2}{*}{9} & 0.0009 & 0.0002 & 0.0015 & 0.0184 & 0.0952 & 0.0657 \\
& $\mathbf{0 . 8}$ & $\mathbf{0 . 2}$ & $\mathbf{0 . 2}$ & $\mathbf{3 . 3}$ & $\mathbf{5 . 9}$ & $\mathbf{5 . 2}$ \\
\hline \multirow{2}{*}{10} & 0.0011 & 0.0003 & 0.0023 & 0.0187 & 0.0937 & 0.0660 \\
& $\mathbf{1 . 0}$ & $\mathbf{0 . 3}$ & $\mathbf{0 . 3}$ & $\mathbf{3 . 4}$ & $\mathbf{5 . 9}$ & $\mathbf{5 . 2}$ \\
\hline \multirow{2}{*}{11} & 0.0136 & 0.0127 & 0.0527 & 0.0920 & 0.2547 & 0.1944 \\
& $\mathbf{1 2 . 2}$ & $\mathbf{1 3 . 6}$ & $\mathbf{8 . 0}$ & $\mathbf{1 6 . 6}$ & $\mathbf{1 5 . 9}$ & $\mathbf{1 5 . 5}$ \\
\hline \multirow{2}{*}{12} & 0.0135 & 0.0130 & 0.0438 & 0.0848 & 0.2577 & 0.1982 \\
& $\mathbf{1 2 . 1}$ & $\mathbf{1 3 . 9}$ & $\mathbf{6 . 6}$ & $\mathbf{1 5 . 3}$ & $\mathbf{1 6 . 1}$ & $\mathbf{1 5 . 8}$ \\
\hline \multirow{2}{*}{12} & & & & & &
\end{tabular}

Next, the contribution of the slip systems at the position 5 and 6 in Fig. 5 was studied. By comparing the relative contributions of the various slip systems to the overall shear strain at complete unloading, a significant difference in the prediction with EMSGCP and CP at position 5 was noticed (Table 5). However, at position 6 the difference was minimal, which reflects in the identical lattice rotation magnitudes in Fig. 5. Thus, from our study it can be concluded that a direct relationship exists between the lattice spins predicted by the CP and the EMSGCP theories and the corresponding contributions of the individual slip systems to the overall deformation. This can be also inferred when the evolution of relative contributions of various slip systems to the overall shear at position 1 along path CD (Table 4) and the respective development of lattice rotations (Fig. 5 (a, c)) are compared. For instance, at half-loading, as the strain gradients are small in magnitude, relative contributions of different slip systems to the overall deformation are almost identical to those for the CP theory, whereas at full loading with a significantly increased strain gradients, different relative contributions were 
observed for different slip systems resulting in different texture evolutions in the EMSGCP and CP theories.

Figure 7 demonstrates the gradient of shear strain $\left(\nabla \gamma^{\beta}\right)$ and effective GND density $\left(n_{G}^{\alpha}\right)$ for different slip systems in (-1 01 1) plane of $\left(\begin{array}{lll}0 & 010\end{array}\right)$ orientation at spatial position 1 for different stages ( $t=0.25 \mathrm{~s}, 0.5 \mathrm{~s}, 0.75 \mathrm{~s}, 1.0 \mathrm{~s})$ of the loading process. To make this plot clear, each line represents the average value for the magnitudes of the two successive slip systems with their magnitudes very close to each other. The plot demonstrates the effect of strain gradients at individual slip system on the overall deformation characteristics. It was observed that the major shear strain gradients occur in slip systems 1, 2, 11 and 12. The fact that the gradient magnitude increases with an increase in the imposed macroscopically inhomogeneous deformation is not surprising. However, the character of the evolution of effective GND density on slip systems (shown in Fig. 7 (b)) does not demonstrate any obvious trend with specific $\nabla \gamma^{\beta}$ on the slip system. This shows that the $n_{G}$ value for any slip system ( $n_{G}^{\alpha}$ ) is affected strongly by the straingradient contributions from all the slip systems (Eq. (7)).

Table 5 


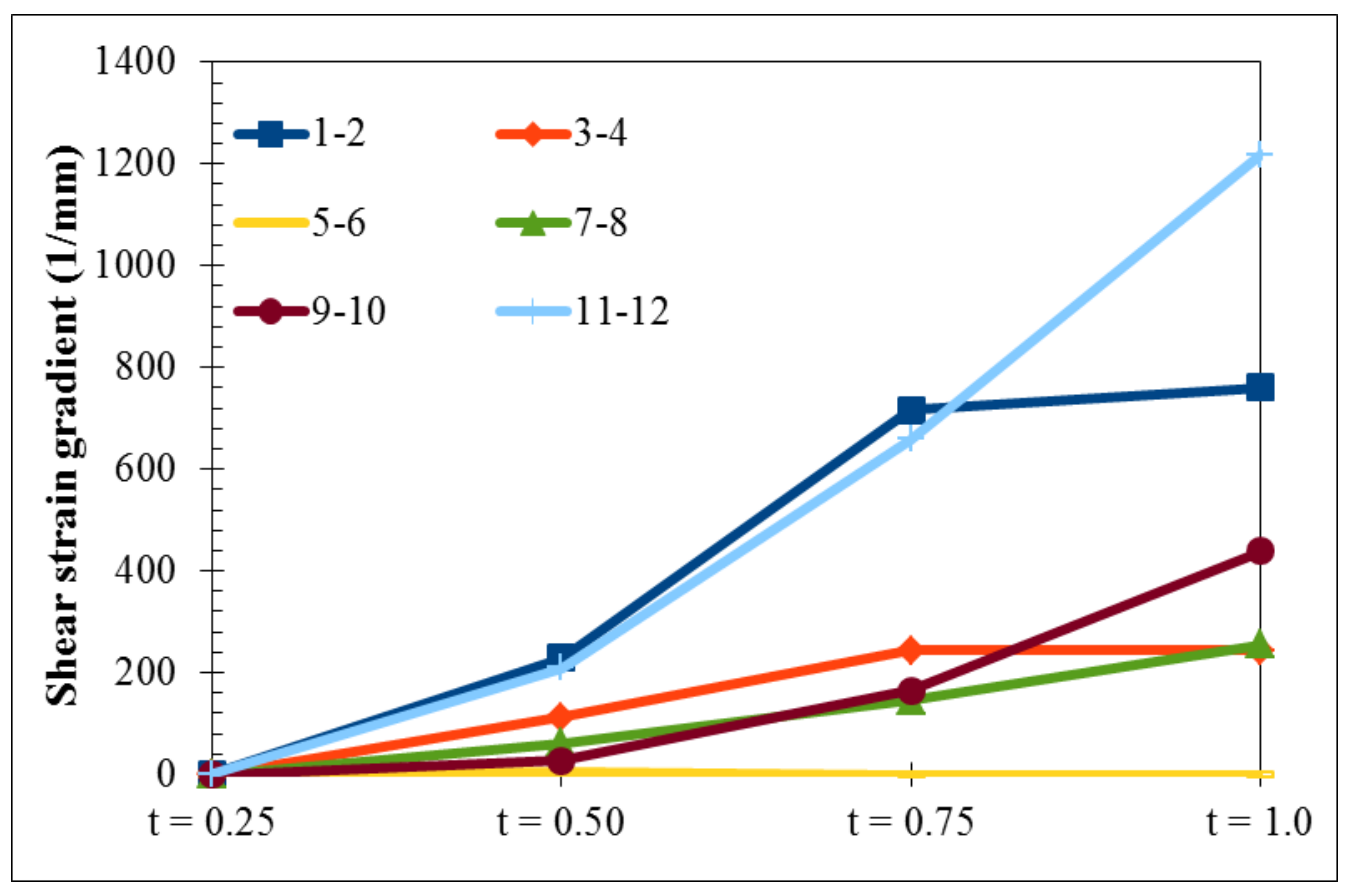

(a)

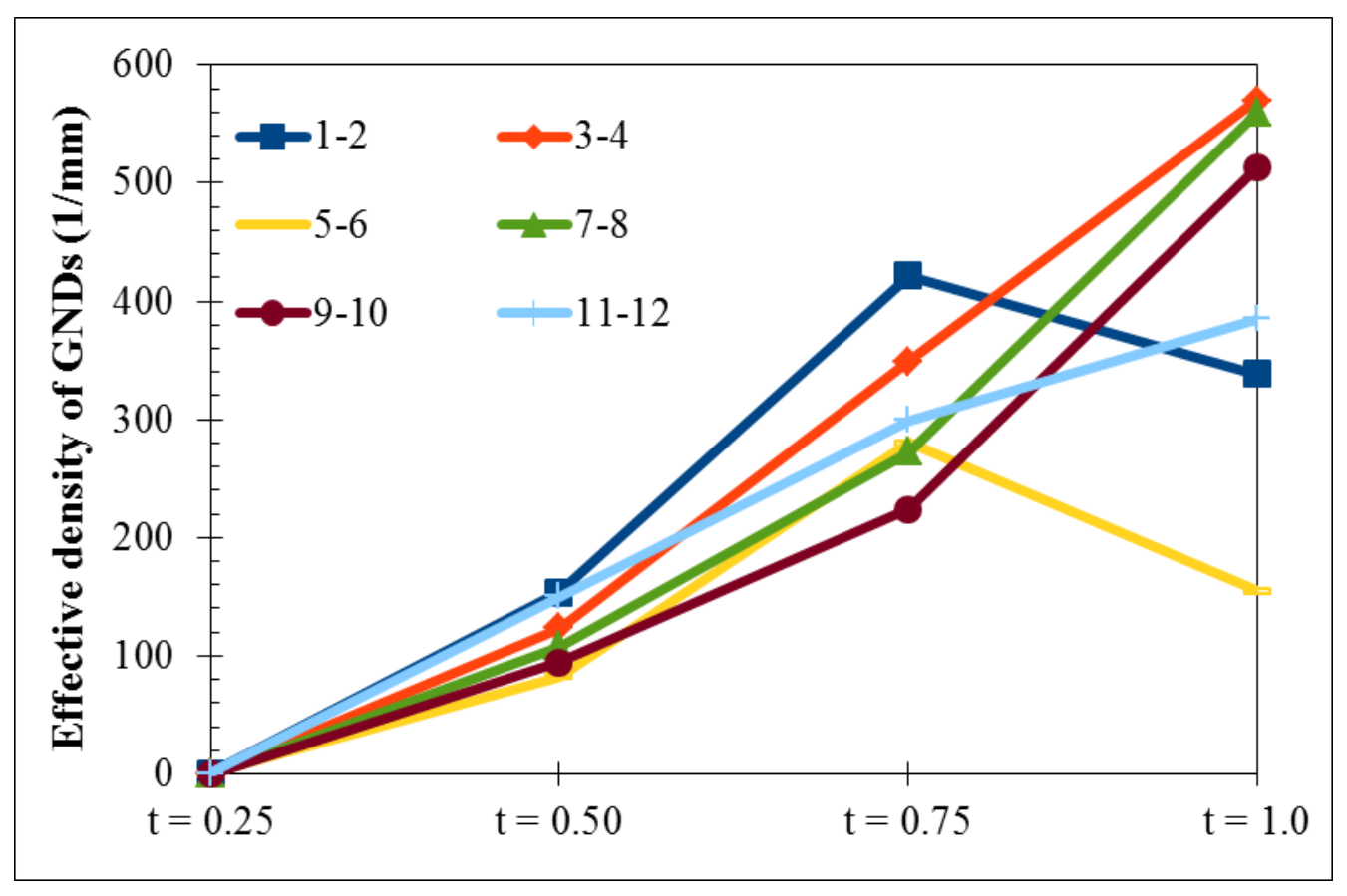

(b)

Fig. 7: Gradient of shear strain $\left(\nabla \gamma^{\beta}\right)$ (a) and effective density of GNDs $\left(n_{G}^{\alpha}\right)$ (b) for different slip systems in (-1 0 1) plane of (010) orientation for position 1 at different loading steps 
The distribution of strain gradients at the cross-section of (-1 0 1) plane for the indented (010) surface was also analysed to investigate a possible relationship with the observed deformation pattern. The distribution of residual GND density in the material after indentation is shown in Fig. 8 and analysed along the path C-D in Fig. 9. It was observed that the distribution of strain gradients in region B showed a double-well pattern similar to the previously observed patterns for deformationinduced lattice rotations (Fig. 4). The strain gradient at position 2 on path C-D is larger than that at position 1 (Fig. 9). It should be noted that at position 2 both the CP and the EMSGCP theories predicted similar shear activity of different slip systems. From this observation, it can be concluded that a presence of strain gradients at a material point does not necessarily entail additional deformations when compared to the conventional CP theory.

\section{Fig. 8}

It was found that a high strain gradient at a material point does not necessarily imply decreased local lattice rotations, when compared to predictions with the CP theory. For instance, the strain gradient at position 2 was higher than that at position 1 (Fig. 9); however, the predictions for lattice rotation with $\mathrm{CP}$ and EMSGCP show minimal difference at position 2, with a noticeable difference at position 1 (Fig. 5 (d)).

\section{Fig. 9}

Fig. 10 shows the distributions of the resulting von Mises stress on the $\left(\begin{array}{lll}-1 & 0 & 1\end{array}\right)$ plane obtained with the two theories. It is clear, that the stress distributions are different. As strain gradients are intrinsic to nanoindentation, the EMSGCP theory accounting for the GNDs represented the deformation patterns and stress distributions more accurately.

\section{Fig. 10}

\section{Concluding remarks}

In this paper, the texture evolution of the Ti alloy single crystal in nano-indentation for different crystallographic orientations was investigated using an enhanced 
model of the strain-gradient crystal-plasticity (EMSGCP). Our study demonstrated that there existed a strong correlation between the patterns of lattice spins and the indents' pile-up profiles. It was noted that the deformation-induced lattice rotations can be predicted more accurately using the strain-gradient crystal-plasticity theory since the effect of GNDs were accounted for.

From our study, the following non-trivial observations were made:

1. The introduction of strain gradients alters the activity of the slip systems and the relative contribution to the overall plastic slip. The EMSGCP theory predicts that plasticity occurs due to activity of multiple slip systems when compared to that of CP theory.

2. The relation between strain-gradient contribution in a particular slip system and the GND density is non-trivial.

3. The presence of a high strain gradient does not necessarily imply increased/decreased lattice rotations.

4. The stress fields predicted with the EMSGCP theory are physical and reflect the true distribution of stresses in the material.

5. In predictions with the strain-gradient theory, the pile-up profile and the lattice rotations are close to those in physical experiments.

\section{Acknowledgement}

The research leading to these results received funding from the European Union Seventh Framework Programme (FP7/2007-2013) under grant agreement No. PITN-GA-2008-211536, project MaMiNa and KAUST baseline fund. MD thanks Prof. Fionn Dunne for useful discussions.

\section{References}

[1] B.N. Lucas, W.C. Oliver, Metall. Mater. Trans. A 30 (1999) 601-610.

[2] Y. Huang, S. Qu, K.C. Hwang, M. Li, H. Gao, Int. J. Plast. 20 (2004) 753-782.

[3] S. Qu, Y. Huang, G.M. Pharr, K.C. Hwang, Int. J. Plast. 22 (2006) 1265-1286. 
[4] N. Zaafarani, D. Raabe, R.N. Singh, F. Roters, S. Zaefferer, Acta Mater. 54 (2006) 1863-1876.

[5] W. Yang, B.C. Larson, G.M. Pharr, G.E. Ice, J.D. Budai, J.Z. Tischler, W. Liu, J. Mater. Res. 19 (2004) 66-72.

[6] S.J. Lloyd, A. Castellero, F. Giuliani, Y. Long, K.K. McLaughlin, J.M. MolinaAldareguia, N.A. Stelmashenko, L.J. Vandeperre, W.J. Clegg, Proceedings of the Royal Society A: Mathematical, Physical and Engineering Science 461 (2005) 2521-2543.

[7] K.K. McLaughlin, N.A., Stelmashenko, S.J. Lloyd, L.J. Vandeperre, W.J. Clegg. Mater. Res. Soc. Symp. Proc. 841 R 1 (2004), 3-1.

[8] G. Winther, Mater. Sci. Eng. A 483 (2008) 40-46.

[9] G.Y. Deng, C. Lu, L.H. Su, X.H. Liu, A.K. Tieu. Mater. Sci. Eng. A 534 (2012) 68-74.

[10] Y. Wang, D. Raabe, C. Klüber, F. Roters, Acta Mater. 52 (2004) 2229-2238.

[11] N. Zaafarani, D. Raabe, F. Roters, S. Zaefferer, Acta Mater. 56 (2008) 31-42.

[12] A. Acharya, A. Beaudoin, J. Mech. Phys. Solids 48 (2000) 2213-2230.

[13] N.A. Fleck, J.W. Hutchinson, J. Mech. Phys. Solids 49 (2001) 2245-2271.

[14] R.K. Abu Al-Rub, G.Z. Voyiadjis, Int. J. Plast. 20 (2004) 1139-1182.

[15] M.E. Gurtin, L. Anand. Int. J. Plast. 21 (2005) 2297-2318.

[16] D.J. Bammann, Mater. Sci. Eng. A 309 (2001) 406-410.

[17] H. Gao, Y. Huang, Scr. Mater. 48 (2003) 113-118.

[18] N.A. Fleck, G.M. Muller, M.F. Ashby, J.W. Hutchinson, Acta Metallur. Mater. 42 (1994) 475-487.

[19] J. Stölken, A. Evans, Acta Mater. 46 (1998) 5109-5115.

[20] N.A Stelmashenko, M.G. Walls, L.M. Brown, Yu V. Milman. Acta Metal. Mater. 41 (1993) 2855-2865. 
[21] W.B. Lee, Y.P. Chen, Int. J. Plast. 26 (2010) 1527-1540.

[22] Z. Zong, J. Lou, O.O. Adewoye, A.A. Elmustafa, F. Hammad, and W.O. Soboyejo, Mater. Sci. Eng. A 434 (2006): 178-187.

[23] M. Demiral, Enhanced gradient crystal-plasticity study of size effects in b.c.c. metal, Loughborough University, UK, 2013 (Ph.D. thesis)

[24] M. Demiral, A. Roy, V.V. Silberschmidt, Comput. Mater. Sci. 79 (2013) 896902.

[25] D. Peirce, R.J. Asaro, A. Needleman, Acta metallurgica 30 (1982) 1087-1119.

[26] K.S. Cheong, E.P. Busso, A. Arsenlis, Int. J. Plast. 21 (2005) 1797-1814.

[27] A. Siddiq, S. Schmauder, Y. Huang, Int. J. Plast. 23 (2007) 665-689.

[28] C. Han, H. Gao, Y. Huang, W.D. Nix, J. Mech. Phys. Solids 53 (2005) 11881203.

[29] K. Nowag, Deformation mechanisms of hard to machine metal Alloys at the micro scale. EMPA, Switzerland - TU Braunschweig, Germany, 2013 (Ph.D. thesis)

[30] T. Altebaeumer, B. Gotsmann, A. Knoll, G. Cherubini, U. Duerig, Nanotech. 19 (2008) 475301.

[31] Y. Liu, S. Varghese, J. Ma, M. Yoshino, H. Lu, R. Komanduri, Int. J. Plast. 24 (2008) 1990-2015.

[32] R. Smith, D. Christopher, S. Kenny, A. Richter, B. Wolf, Phys. Rev. B 67 (2003) 245405. 


\section{Figure captions}

Fig. 1: Pile-up profiles of indented (0.641 0.078 0.764) surface along a path obtained from experiment and simulation at complete unloading [24]

Fig. 2: Rotation angles and rotation directions predicted by FE simulations of indentation after unloading for different crystallographic orientations. Top row:

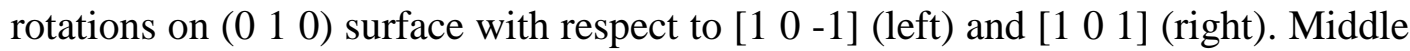
row: rotations on $\left(\begin{array}{lll}1 & 0 & 1\end{array}\right)$ surface with respect to [0 $\left.\begin{array}{lll}0 & 0\end{array}\right]$ (left) and [ $\left[\begin{array}{lll}-1 & 0 & 1\end{array}\right]$ (right). Bottom row: rotations on (1 11 1) surface with respect to [1 $\left.\begin{array}{lll}1 & -2 & 1\end{array}\right]$ (left) and [-1 01 1] (right)

Fig. 3: Pile-up structures of imprints obtained with FE simulations for different crystallographic orientations after unloading: (a) (010) surface; (b) (101) surface; (c) (111) surface

Fig. 4: Distribution of lattice rotations in $\left(\begin{array}{lll}-1 & 0 & 1\end{array}\right)$ plane of $(010)$ orientation obtained with FE simulations using (a) EMSGCP theory and (b) CP theory at complete unloading

Fig. 5: 
Fig. 6: Shear-strain values of different slip systems at spatial positions 1 (a) and 2 (b) along path C-D (see Fig. 4) in (-1 0 1) plane of (010) orientation at complete unloading

Fig. 7:

Fig. 8: Distribution of total effective density of GNDs $\left(\sum_{\alpha=1}^{N} n_{G}^{\alpha}\right)$ in $\left(\begin{array}{lll}-1 & 0 & 1\end{array}\right)$ plane of (010) orientation after unloading

Fig. 9: Total effective density of GNDs $\left(\sum_{\alpha=1}^{N} n_{G}^{\alpha}\right)$ along path C-D (see Fig. 4) in (-1 0 1) plane of (010) orientation after unloading

Fig. 10: Distribution of von Mises stress in (- $\left.\begin{array}{lll}-1 & 0 & 1\end{array}\right)$ plane of (010) orientation at complete unloading obtained with FE simulations using (a) EMSGCP theory and (b) CP theory

\section{Table captions}

Table 1: Available slip systems for Ti alloy single crystal for $\{112\}<111>$ set

Table 2: Material parameters for Ti-alloy single-crystal micropillars used in the model of micropillar-compression experiment (Demiral et al., 2013)

Table 3: Lattice rotations at points along path C-D and E-F at complete unloading (see Fig. 4) using CP and EMSGCP theories

Table 4:

Table 5: Magnitudes of relative shear strain of different slip systems (in \%) at positions 5 and 6 along path E-F (see Fig. 4) in (-1 0 1) plane of (010) orientation at complete unloading 